\title{
PREVENÇÃO DO CONSUMO DE DROGAS ENTRE ESCOLARES: RELATOS MEDIADOS PELA EXTENSÃO UNIVERSITÁRIA
}

DRUG USE PREVENTION AMONG SCHOOL STUDENTS: REPORTS MEDIATED BY UNIVERSITY OUTREACH

PREVENCIÓN DEL CONSUMO DE DROGAS ENTRE ESCOLARES: INFORMES MEDIADOS POR LA EXTENSIÓN UNIVERSITARIA

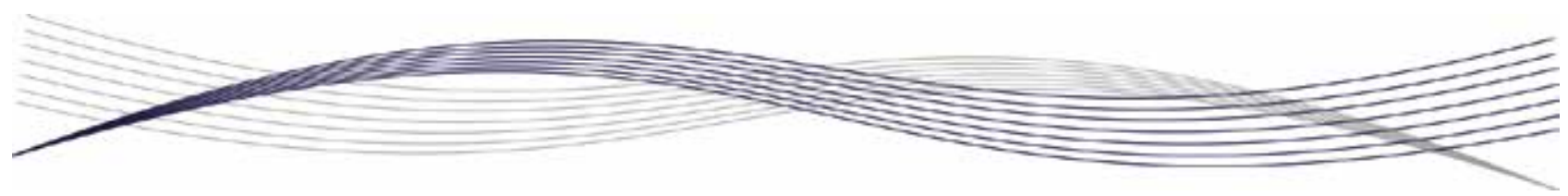

Palavras-chave: Drogas; Extensão Universitária; Escola Pública; Prevenção.

\section{Keywords:}

Drugs; University Outreach; Public School; Prevention.

Palabras clave: Drogas; Extensión Universitaria; Escuela Pública; Prevención.

Submetido: $17 / 04 / 2018$

Aprovado: $26 / 10 / 2018$

Autor(a) para Correspondência: Alessandra Maria C. de Sousa R. Napoleão Lima, 1281, apto. 101 Jockey Clube - Teresina-PI 64049-220

E-mail: alecerqueiramed@gmail.com

\section{RESUMO}

A extensão universitária é um dos pilares do Ensino Superior brasileiro, em virtude tanto da possibilidade de aproximação entre a universidade $e$ a sociedade como da produção de conhecimento entre os extensionistas $e$ o agrupamento social. Nesse contexto, é inquestionável o relevante papel da escola no desenvolvimento sadio dos alunos, já que contribui com a formação global do jovem e da sociedade. Este estudo abordou o tema drogas, no contexto escolar, sob a perspectiva de um trabalho de prevenção de seu consumo. Diante da necessidade de atenuar esse problema de saúde pública, o projeto de extensão universitária "Drogas: Informar para Prevenir" foi realizado de 2015 a 2017. Seu propósito foi promover ações de conscientização voltadas à prevenção do consumo de drogas, de modo a gerar discussões e reflexões acerca do tema drogas entre estudantes de $8^{a}$ e $g^{a}$ série em uma escola municipal de Teresina-PI. Adotou-se o formato de oficinas (com uso de diversos recursos) no processo de construção das vivências de prevenção primária ao uso indevido de substâncias psicoativas. Este artigo relata os resultados obtidos junto aos escolares.

\footnotetext{
1. Dentista. Doutora em Clínicas Odontológicas pela Faculdade São Leopoldo Mandic. Professora da Universidade Estadual do Piauí (UESPI). Teresina (PI), Brasil. E-mail: lutolstenko@hotmail.com

2. Médica graduada pela UESPI. Teresina (PI), Brasil. E-mail: alecerqueiramed@gmail.com

3. Aluno de Graduação em Medicina na UESPI. Teresina (PI), Brasil. E-mail: matheus.vvp@hotmail.com
} 


\section{ABSTRACT}

University outreach is one of the Brazilian Higher Education pillars, both due to the possibility of getting university closer to society and producing knowledge between university outreach participants and social grouping. In this context, the relevant role played by school in the students' healthy development is indisputable, because this contributes to the overall education of a young person and a society. This study addressed drugs as a theme, in the school context, from the perspective of a work to prevent its consumption. Faced with the need to mitigate this public health issue, the university outreach project "Drugs: Inform to Prevent" was conducted from 2015 to 2017. Its purpose was promoting awareness-raising actions aimed at preventing drug use, in order to generate discussions and exchange of ideas by having drugs as a theme among $8^{\text {th }}$ and $9^{\text {th }}$ grade students at a municipal school in Teresina, Piaui, Brazil. The workshop format (using a variety of resources) was adopted in the process of constructing experiences for primary prevention of psychoactive substance abuse. This article reports the results obtained along with school students.

\section{RESUMEN}

La extensión universitaria es uno de los pilares de la Educación Superior brasileña, tanto por la posibilidad de acercar la universidad a la sociedad como por la producción de conocimiento entre los participantes de la extensión universitaria y el agrupamiento social. En este contexto, el relevante papel que juega la escuela en el desarrollo saludable de los estudiantes es indiscutible, ya que contribuye a la educación global de un joven y una sociedad. Este estudio abordó el tema drogas, en el contexto escolar, desde la perspectiva de un trabajo de prevención de su consumo. Ante la necesidad de atenuar este problema de salud pública, el proyecto de extensión universitaria "Drogas: Informar para Prevenir" se llevó a cabo de 2015 a 2017. Su propósito fue promover acciones de concienciación dirigidas a la prevención del consumo de drogas, para generar discusiones y reflexiones acerca del tema drogas entre estudiantes de $8^{a}$ y $9^{a}$ serie en una escuela municipal en Teresina, Piaui, Brasil. El formato del taller (utilizando una variedad de recursos) fue adoptado en el proceso de construcción de experiencias para la prevención primaria del abuso de sustancias psicoactivas. Este artículo informa sobre los resultados obtenidos junto con los escolares.

\section{INTRODUÇÃO}

A extensão universitária, um dos pilares do Ensino Superior brasileiro (junto com o ensino e a pesquisa), preenche lacunas ao tornar os conhecimentos científicos produzidos na academia acessiveis à sociedade. Nesse sentido, representa uma forma de democratização do conhecimento e possibilita o estabelecimento de diálogo com as comunidades, facultando aos extensionistas a aquisição de saber em relação a situações antes desconhecidas ${ }^{1}$.

0 consumo de drogas é considerado um problema de saúde pública, não somente em função de sua alta frequência, mas principalmente devido aos malefícios com consequências biopsicossociais observados entre os jovens e na sociedade como um todo ${ }^{2}$. A partir da década de 1970, quando surgiu a estratégia de redução do uso indevido de drogas, a Organização das Nações Unidas para a Educação, a Ciência e a Cultura (Unesco) passou a ressaltar a abordagem preventiva a abuso de drogas, tendo a escola como meio principal para tal processo ${ }^{3}$.

0 Estatuto da Criança e do Adolescente (ECA) constituiu um avanço legal fundamental para as crianças e os adolescentes como pessoas em desenvolvimento. 0 ECA trouxe ao Brasil uma nova visão acerca desses sujeitos, incluindo-os em um período específico dos ciclos de desenvolvimento, o que garante prioridade absoluta na elaboração e aplicação de políticas que assegurem a efetivação de seus direitos referentes a vida, saúde, alimentação, educação, busca de identidade, crenças, valores e lugar no espaço social ${ }^{4}$.

A escola assume papel de destaque no desenvolvimento sadio do adolescente e do adulto, pois contribui para a formação global do jovem e da sociedade ${ }^{5}$. Desse modo, sua função na prevenção do consumo de drogas é educar as crianças e os jovens a buscar e desenvolver sua identidade e subjetividade, promover e integrar sua educação intelectual e emocional e incentivar a cidadania e a responsabilidade social, bem como garantir que eles incorporem hábitos saudáveis em seu cotidiano. Surge, assim, a noção de escola como unidade promotora de saúde ${ }^{6}$.

Vale ressaltar que a escola constitui local privilegiado para trabalhar a prevenção às drogas 
tanto por proporcionar acesso aos jovens como por sua natureza educacional. No entanto, o despreparo do corpo docente para lidar com ações de educação em saúde junto aos alunos no que tange ao consumo de substâncias psicoativas é uma realidade inerente ao contexto escolar. Apesar do aumento do uso de tais substâncias ao longo dos anos, infelizmente, não se observou uma mudança correspondente nas políticas públicas capaz de minimizar seu impacto. Diante disso, a escola demanda cada vez mais ações e propostas voltadas à educação em saúde, com vistas a atender ao princípio da integralidade do direito à saúde do Sistema Único de Saúde (SUS).

0 projeto de extensão universitária “Drogas: Informar para Prevenir" se situa nesse âmbito, uma vez que o tema drogas envolve discussões alinhadas com a Constituição Federal de 1988 diante de decisões relativas ao corpo e à saúde ${ }^{7}$.

Esse projeto se justifica ao considerar os dados epidemiológicos que retratam a problemática do uso de drogas no país. De acordo com o $2^{\circ}$ Levantamento Nacional de Álcool e Drogas (Lenad), da Universidade Federal de São Paulo (Unifesp), o Brasil é $2^{\circ}$ maior consumidor mundial de cocaína e derivados: mais de 6 milhões de pessoas já experimentaram essas substâncias ao longo da vida e quase metade dos usuários (45\%) provaram a(s) droga(s) em questão antes dos 18 anos. 0 Lenad indicou o Nordeste como a região com maior consumo de cocaína e derivados $(40 \% \text { do total })^{8}$.

Segundo dados do Centro Brasileiro de Informações sobre Drogas Psicotrópicas (Cebrid), Teresina-PI é a terceira capital do país em termos de consumo excessivo de álcool. E, de acordo com - $6^{\circ}$ Levantamento Nacional sobre o Consumo de Drogas Psicotrópicas, $63,4 \%$ dos estudantes da rede pública e privada de ensino das 27 capitais brasileiras relataram contato com o álcool ${ }^{9}$.

Pensando em um plano de ação conjunta voltado ao tema drogas, o projeto de extensão universitária foi realizado de 2015 a 2017 por acadêmicos de Medicina da Universidade Estadual do Piauí (UESPI).

Este artigo relata os resultados obtidos a partir das vivências de prevenção primária ao uso indevido de substâncias psicoativas entre escolares.

\section{METODOLOGIA}

Com o propósito de adotar uma abordagem diferenciada à prevenção ao consumo de drogas, optou-se por uma metodologia participativa e

\section{... $63,4 \%$ dos estudantes \\ da rede pública e privada de ensino [..] relataram contato com o álcool.}

problematizadora. Isso suscitou reflexão sobre os danos causados pelas substâncias psicoativas e despertou o interesse em analisar esse quadro de escolhas equivocadas que afeta os jovens brasileiros cada vez mais precocemente.

0 projeto de extensão universitária "Drogas: Informar para Prevenir" vinculado ao Curso de Medicina da UESPI (Campus Torquato Neto) e realizado de 2015 a 2017 com alunos de $8^{a}$ e $9^{\text {a }}$ série em Teresina não enfocou apenas os males causados pelas drogas. Buscou-se demonstrar que a vida tem muito mais a oferecer para evitar o primeiro contato do jovem com esse mundo por meio do método de problematização e da organização de oficinas acerca do tema drogas.

A prevenção às drogas norteou todo 0 desenvolvimento do projeto, que recorreu a estratégias diversificadas, como seminários, palestras, jornais, desenhos e práticas de expressão corporal. Com base em princípios de arte-educação, buscou-se promover a sensibilização e participação no âmbito da escola pública, para conscientizá-la sobre a importância do não uso de drogas.

A primeira fase, "Implantação do projeto: espaço e equipe", consistiu na escolha do espaço para as atividades, após contato e consentimento dos responsáveis pelo local do estudo, além da seleção de estudantes de $8^{a}$ e $9^{a}$ série para participar na execução do projeto.

A partir da definição do espaço e da formação da equipe do projeto, a segunda fase, "Investigação bibliográfica", buscou ampliar a capacitação teórico-científica dos participantes diante de questões relacionadas à prevenção do consumo de drogas. Adotou-se como procedimento a busca, nos principais bancos de dados virtuais, de artigos científicos e de livros que abordam o tema drogas, além de orientações de profissionais que atuam na área que abrange esse tema.

A terceira fase, preparação/planejamento das atividades, contou com palestras sobre o uso abusivo de drogas. 0 conteúdo abarcou figuras e vídeos, 
buscando dimensionar junto aos escolares os efeitos maléficos das substâncias psicoativas, de modo a gerar discussões e reflexões com embasamento científico. Todos os materiais apresentados continham endereço de e-mail para contato, caso alunos, professores e o público geral necessitassem de maiores esclarecimentos.

A quarta fase foi a execução do projeto. A equipe se deslocou para o local do estudo nas datas agendadas em comum acordo com a instituição pública de Ensino Fundamental, na zona urbana de Teresina. 0s encontros semanais envolveram palestras, grupos de discussão, debates e dinâmicas.

Adotou-se o seguinte conteúdo programático:

- "Conceitos e abordagens sobre drogas e prevenção";

- “Construção de hábitos saudáveis e valores sociais";

- "Diferentes relações com as drogas: abordagens na adolescência";

- "Drogas: fatores de risco e fatores de proteção";

- "Prevenção ao uso de álcool e seu impacto na adolescência";

- "Maconha, crack, cocaína e derivados: nem pensar!"; e

- "Prevenção ao uso do tabaco: respire essa ideia!".

Foram utilizados tanto recursos eletrônicos (DVD, projetor, notebook e televisor) como materiais impressos para a elaboração de exercícios e as atividades de leitura. Após as palestras, abriu-se um período para a discussão de dúvidas dos escolares. As dinâmicas foram desenvolvidas para promover a interação entre os participantes, de modo que conversassem com naturalidade, sem cobranças, sobre temas que causam certo constrangimento. 0s grupos de discussão foram formados após a exibição de filme ou documentário ou após a análise de entrevistas e reportagens.

Discutiram-se os diferentes tipos de drogas e os diversos efeitos fisiológicos decorrentes do consumo dessas substâncias, enfatizando os grupos de drogas e as modificações que causam no funcionamento do cérebro. Todas as oficinas foram realizadas na própria instituição de ensino à qual os escolares estão vinculados, ou seja, na escola que frequentam.

Ao término de cada atividade nas oficinas de discussão, os participantes relataram sua

\author{
0 abuso é o \\ consumo excessivo \\ que repercutirá \\ patologicamente \\ no individuo.
}

aprendizagem em debates e esclareceram dúvidas com os extensionistas, compartilhando experiências, tanto pessoais como do âmbito familiar, acerca do tema drogas.

\section{RESULTADOS E DISCUSSÃO}

A primeira unidade temática das oficinas foi "Conceitos e abordagens sobre drogas e prevenção", com conceitos como abuso, dependência e abstinência de drogas. A discussão se pautou pela apresentação de vídeos sobre os tipos de drogas e seus principais efeitos maléficos na vida escolar, familiar e comunitária. As formas de consumo de drogas são classificadas em uso, abuso e dependência termos geralmente utilizados como sinônimos, apesar de sua especificidade.

Por uso se entende o consumo esporádico de determinada droga. 0 abuso é o consumo excessivo que repercutirá patologicamente no indivíduo. Já a dependência química é o resultado do aumento da tolerância à substância (o corpo do indivíduo se adapta à ação da droga), fazendo com que só se alcance a sensação da dose inicial quando há consumo de altas doses dessa droga. Ao término das atividades houve debate entre alunos, extensionistas e professores, adequando as oficinas aos pressupostos da prática extensionista por meio de via de mão dupla trânsito de saberes entre a universidade e a sociedade.

Já no primeiro encontro se percebeu a realidade do uso de drogas lícitas e ilícitas pelos alunos. No segundo encontro, as atividades se concentraram em relatar as consequências e os males decorrentes do uso prolongado de drogas, enfatizando que "o melhor é prevenir". Demonstrou-se aos alunos o que ocorre em nosso sistema nervoso com o uso de drogas psicotrópicas, sempre interagindo com os participantes. Após o contato inicial, a apresentação de vídeos sobre a realidade de quem usa drogas estimulou a discussão, levando os alunos a perguntar e dividir suas experiências. 
A segunda unidade temática das oficinas foi "Construção de hábitos saudáveis e valores", onde os escolares expuseram suas opiniões sobre quais hábitos saudáveis consideram estar presentes em seu cotidiano e os extensionistas esclareceram 0 relevante papel da escola na construção do alicerce para pôr esses hábitos e valores em prática.

A terceira unidade temática das oficinas, “Diferentes relações com as drogas: abordagens na adolescência", elencou diversas situações de risco às quais os escolares estão expostos na sociedade atual e onde e de que maneira eles podem pedir ajuda para enfrentar tais situações. E na quarta unidade temática, "Drogas: fatores de risco e fatores de proteção", os escolares se dividiram em grupos e confeccionaram cartazes listando os fatores de risco do uso de drogas e os fatores de proteção contra as drogas, de acordo com o que aprenderam nas unidades temáticas anteriores.

As três últimas unidades temáticas, "Prevenção ao uso de álcool e seu impacto na adolescência", "Maconha, crack, cocaína e derivados: nem pensar!" e "Prevenção ao uso do tabaco: respire essa ideia!", enfocaram os grupos de drogas, expondo seus principais efeitos maléficos no organismo e apresentando medidas de prevenção, com vistas a alcançar o resultado proposto - evitar o uso de drogas por escolares. Nessas oficinas, as drogas foram classificadas de acordo com os efeitos que causam no sistema nervoso central (depressoras, estimulantes ou perturbadoras). As substâncias depressoras diminuem a atividade cerebral, deixando o indivíduo "desligado". As substâncias estimulantes aumentam a atividade cerebral, deixando o indivíduo "ligado", "elétrico". E o terceiro grupo envolve substâncias que produzem uma mudança qualitativa na atividade cerebral ${ }^{10}$.

Os escolares se mostraram receptivos ao tema drogas na grande maioria das vezes, apesar de não compreenderem a dimensão da problemática do uso de drogas. Percebeu-se, no decorrer dos diálogos, que eles buscaram rever suas concepções sobre as drogas e repassá-las para suas comunidades. 0 consumo de drogas é uma realidade inerente ao contexto escolar e a maior dificuldade observada foi atingir os escolares usuários de substâncias psicoativas.

Uma vez concluídas as atividades do projeto de extensão universitária “Drogas: Informar para Prevenir", os alunos se mostraram capazes de repassar os conhecimentos adquiridos na instituição de ensino para seus ambientes familiares e suas

\author{
O consumo de \\ drogas é uma \\ realidade inerente \\ ao contexto \\ escolar...
}

comunidades. 0s professores e administradores elogiaram o projeto e destacaram a importância de atividades com propósitos preventivos no âmbito da escola pública.

Constatou-se que tanto os alunos como os professores mostraram interesse em dar continuidade às discussões sobre as drogas. Dentre os itens sugeridos, destacou-se o trabalho relativo à autoestima, tendo em vista que a autoestima é crucial para que os as crianças e os adolescentes venham a ser pessoas aptas a lidar com desafios e responsabilidades com segurança.

Percebeu-se que o diálogo ainda é o melhor aspecto da prevenção tanto por parte dos pais como dos professores, considerando a importância do papel do educador na proteção dos escolares contra as drogas. Abordar o tema naturalmente na escola torna mais fácil que os educadores comentem suas suspeitas ou certezas com os pais, já que a escola pode e deve ser uma instituição de ampla inserção social das crianças e dos adolescentes.

Os participantes do projeto de extensão universitária da UESPI combinaram recursos educativos e científicos em suas ações com propósitos preventivos no âmbito da escola pública, articulando o ensino e a pesquisa nas práticas do Ensino Superior. Desse modo, a universidade transcende seus muros e vai até a sociedade, possibilitando que se estabeleça uma inter-relação transformadora.

0 intercâmbio entre os diferentes níveis de ensino tende a promover uma produção do saber decorrente do confronto com a realidade social, o que estimula a democratização do conhecimento e a efetiva participação das comunidades no avanço da ciência.

A maior dificuldade na execução do projeto consistiu em abordar a prevenção às drogas de modo lúdico, tornando conhecimentos científicos acessiveis a participantes que se encontram em uma faixa etária marcada por dilemas éticos, morais e sociais. A maioria dos escolares usuários de drogas 
tende a ver o contato com substâncias psicoativas como um meio de autoafirmação.

Nesta iniciativa, os extensionistas da UESPI tiveram a oportunidade de estabelecer vínculos com os escolares em um espaço aberto ao diálogo e à troca de saberes sobre o consumo de drogas, buscando desenvolver:

- A formação em saúde pautada pela capacitação teórico-científica;

- 0 espírito crítico;

- A ética e cidadania; e

- 0 princípio da indissociabilidade entre ensino, pesquisa e extensão no Ensino Superior.

\section{CONCLUSÃO}

0 tripé ensino, pesquisa e extensão assume cada vez mais importância no Ensino Superior brasileiro, com abordagem de temas de interesse da sociedade como um todo. No setor saúde, a extensão universitária guarda estreita relação com o princípio da integralidade do direito à saúde do SUS. Assim, o projeto de extensão universitária “Drogas: Informar para Prevenir", realizado de 2015 a 2017, constituiu uma iniciativa do Curso de Medicina da UESPI com significativos resultados no âmbito da escola pública, ao abordar um tema tão complexo como as drogas e as repercussões do consumo de substâncias psicoativas junto aos escolares.

As ações de educação em saúde, por meio de dinâmicas que estimularam o desenvolvimento físico, mental, moral, espiritual e social dos participantes, mostraram-se benéficas ao abrir espaço para discussões e reflexões acerca do tema drogas entre estudantes de $8^{\mathrm{a}}$ e $9^{\mathrm{a}}$ série, com vistas à adoção de hábitos saudáveis e diminuição dos índices de uso de drogas entre os escolares.

Nesse contexto, considera-se que o educador assume papel central, visto que tem contato contínuo com os escolares em sua própria instituição de ensino. Cabe ao professor orientar, prevenir e encaminhar os escolares que apresentam uma mudança de comportamento sugestiva de consumo de drogas.

Outras alternativas podem ser adotadas no âmbito da escola pública, a partir da inclusão de orientações sobre o uso de drogas no projeto pedagógico, tendo em vista as necessidades de cada comunidade escolar. Reuniões com periodicidade regular podem motivar os pais e responsáveis a ficar mais atentos a mudanças comportamentais que indiquem o consumo

\section{...considera-se que o educador \\ assume papel \\ central...}

de substâncias psicoativas por parte dos escolares. Tal iniciativa se mostra extremamente importante, pois reuniões dessa natureza proporcionam evidências de que a família está de acordo ou não com a proposta de combate às drogas, além de facilitar a identificação e o tratamento de usuários de drogas na comunidade escolar.

\section{CONTRIBUIÇÃO DOS AUTORES}

Luciana Tolstenko Nogueira contribuiu com 0 delineamento do estudo e a redação e revisão crítica do manuscrito. Alessandra Maria Cerqueira de Sousa contribuiu com a realização da pesquisa, o delineamento do estudo e a redação do manuscrito. Matheus Veras Viana Portela contribuiu com a realização da pesquisa e a redação do manuscrito.

\section{REFERÊNCIAS}

1. Armani D. Como elaborar projetos? Guia prático para elaboração e gestão de projetos sociais. Porto Alegre: Tomo; 2006.

2. Zeitoune RCG, Ferreira VS, Silveira HS, Domingos AM, Maia AC. 0 conhecimento de adolescentes sobre drogas lícitas e ilícitas: uma contribuição para a enfermagem comunitária. Esc Anna Nery Rev Enferm [serial on the internet]. 2012 [cited 2018 Nov 14];16:57-63. Available from: http://www.scielo.br/ pdf/ean/v16n1/v16n1a08.pdf

3. Moreira FG, Silveira DX, Andreolli SB. Situações relacionadas ao uso indevido de drogas nas escolas públicas da cidade de São Paulo. Rev Saúde Pública [serial on the internet]. 2006 [cited 2018 Nov $14] ; 40(5): 810-7$. Available from: http://www.scielo. $\mathrm{br} / \mathrm{pdf} / \mathrm{rsp} / \mathrm{v} 40 \mathrm{n} 5 / 10 . \mathrm{pdf}$

4. Brasil. Lei n. 8.069, de 13 de julho de 1990. Dispõe sobre o Estatuto da Criança e do Adolescente e dá outras providências. Diário Oficial da União, Brasília (1990 Jul 16); Sec 1. 
5. Santos FD, Queiroz AKC, Teófilo ACS, Mesquita HLMA, Viana RS. Combate ao uso de drogas por adolescentes: estímulo a atitudes saudáveis no círculo de cultura. Sanare (Sobral, Online) [serial on the internet]. 2016 [cited 2018 Nov 14];15(2):549. Available from: https://sanare.emnuvens.com.br/ sanare/article/view/1038/584

6. Ferreira MA, Alvim NAT, Teixeira MLO, Veloso RC. Saberes de adolescentes: estilo de vida e cuidado à saúde. Texto Contexto Enferm [serial on the internet]. 2007 [cited 2018 Nov 14];16(2):21724. Available from: http://www.scielo.br/pdf/tce/ $\mathrm{v} 16 \mathrm{n} 2 / \mathrm{a} 02 \mathrm{v} 16 \mathrm{n} 2$

7. Pedroso RS, Oliveira MS, Araújo RB, Castro MG, Melo WV. Expectativas de resultados frente ao uso de álcool, maconha e tabaco. Rev Psiquiatr Rio Gd Sul [serial on the internet]. 2006 [document on the internet];28(2):198-206. Available from: http:// www.scielo.br/pdf/rprs/v28n2/v28n2a12.pdf

8. Instituto Nacional de Ciência e Tecnologia para Políticas Públicas de Álcool e Outras Drogas. II Levantamento Nacional de Álcool e Drogas. São Paulo: Unifesp; 2012.

9. Carlini ELA, Noto AR, Sanchez ZVDM, Carlini CMA, Locatelli DP, Abeid LR, et al. VI Levantamento Nacional sobre o Consumo de Drogas Psicotrópicas entre Estudantes do Ensino Fundamental e Médio das Redes Pública e Privada de Ensino nas 27 Capitais Brasileiras. Brasília, DF: Senad; 2010.

10. Almeida Filho AJ, Ferreira MA, Gomes MLB, Silva RC, Santos TCF. 0 adolescente e as drogas: consequências para a saúde. Esc Anna Nery Rev Enferm [serial on the internet]. 2007 [cited 2018 Nov 14];11(4):605-10. Available from: http://www. scielo.br/pdf/ean/v11n4/v11n4a08
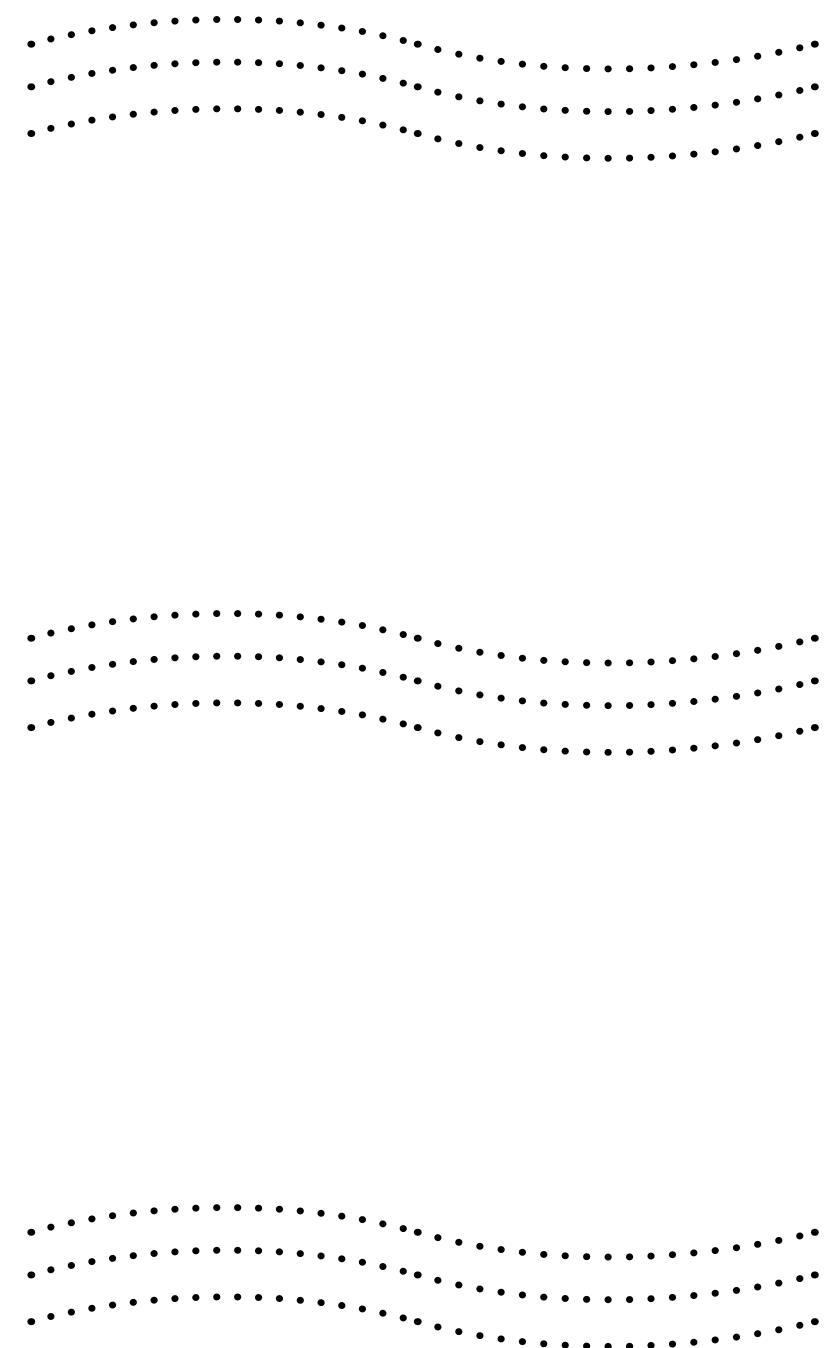
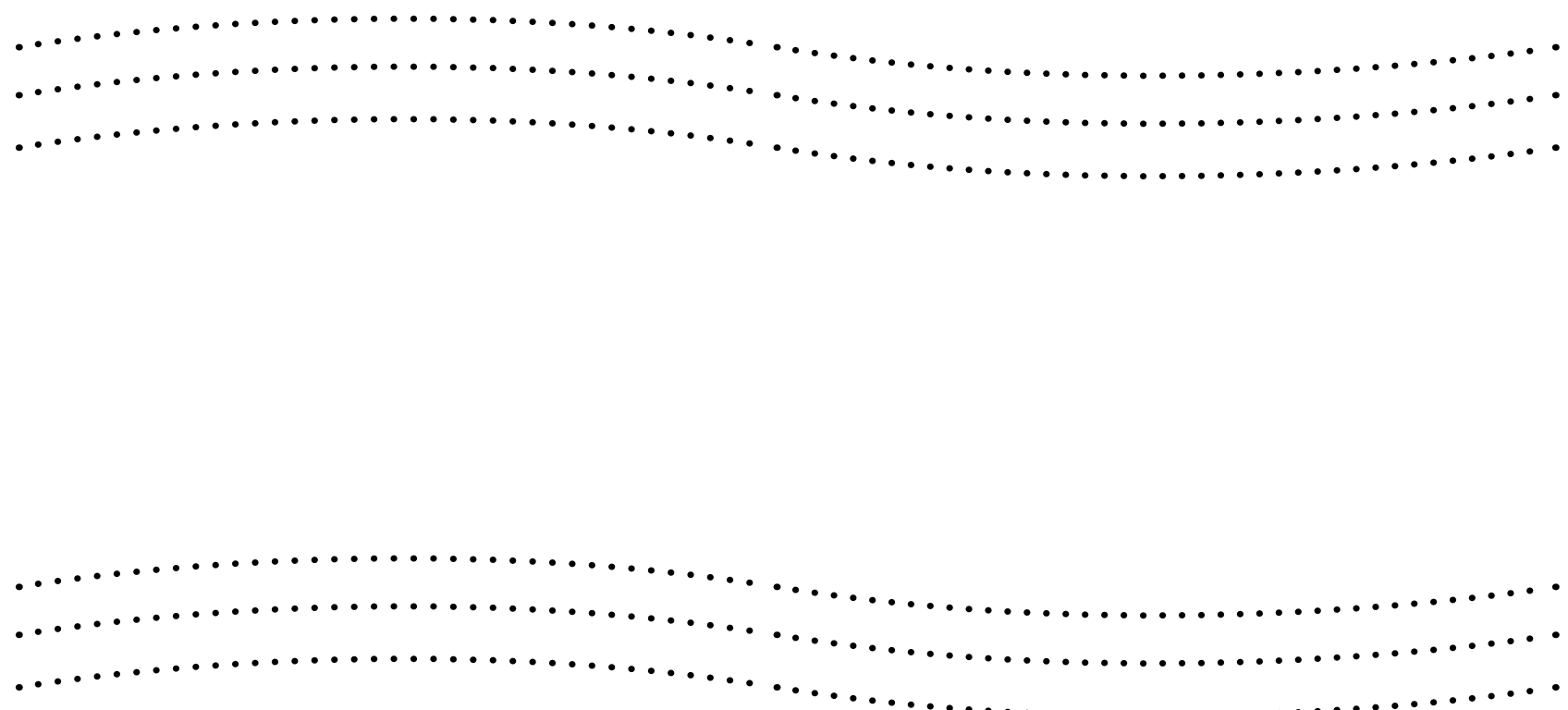